Baca, A.M., León, M.E., Mayta, J. \& Bancayán, C. (2014). Aseguramiento de la calidad de la formación universitaria en el Perú. Revista Electrónica Interuniversitaria de Formación del Profesorado, 17 (3), 3547.

DOI: http://dx.doi.org/10.6018/reifop.17.3.204151

\title{
Aseguramiento de la calidad de la formación universitaria en el Perú
}

Ana Miluzka Baca Gamarra, María Elvira León Di Gianvito, Juan Mayta Carlos, Carlos Bancayán Oré

Universidad de San Martín de Porres

\section{Resumen}

La búsqueda permanente del hombre por entregar productos y servicios de calidad, ha ido evolucionando y pasando del rubro de la empresa hasta los servicios; las universidades, no escapan de este concepto, iniciándose este quehacer en los EEUU de NA, con la aplicación de sus modelos de calidad.

La creación del EEES fortaleció la cooperación entre los estados de la Unión Europea, en el ámbito de la educación superior, permitiéndoles concertar y acordar lineamientos comunes entre las diversas modalidades de sus sistemas educativos.

En Latinoamérica quien inició prácticas de aseguramiento de la calidad de la educación superior fue Chile, en 1990. Posteriormente todos los países de la Región incorporaron este mecanismo en sus sistemas universitarios.

En el Perú existió preocupación por asegurar la calidad de la educación universitaria desde la década del noventa. Se realizaron diversas acciones para superar las deficiencias de calidad existentes. En el año 2006 se concreta la creación del Sistema Nacional de Evaluación, Acreditación y Certificación de la Calidad Educativa, SINEACE.

En diversas universidades peruanas existen experiencias con acreditaciones extranjeras, dirigidas a asegurar la calidad educativa. Un ejemplo es la Universidad de San Martín de Porres, cuya totalidad de carreras han sido acreditadas internacionalmente.

\section{Palabras clave}

Aseguramiento de la calidad; Acreditación; Acreditación en el Perú; Acreditación en Latinoamérica.

Contacto:

Ana Miluzka Baca Gamarra, abacag@usmp.pe, Av. Salaverry № 1136 Jesús María, Lima, Perú. 


\title{
Quality assurance of higher education in Peru
}

\begin{abstract}
The ongoing quest of man by delivering quality products and services has evolved and passing from the sector companies to services; the universities, are not exception to this concept. The beginning of this task was USA, with the implementation of their quality models.
\end{abstract}

The creation of the EEES (Spanish initials) strengthened cooperation between European Union states in the scope of higher education, enabling them to coordinate and accord on common guidelines among different forms of their education systems.

In Latin America Chile started since 1990 practices of quality assurance in higher education. Later, all countries of the region incorporated this works in their university systems.

Since nineties, Peru there was concern to ensure the quality of university education. Different Actions were taken to overcome deficiencies of existing quality. In 2006 was created the National System of Evaluation, Accreditation and Certification of Educational Quality, SINEACE.

In different Peruvians Universities we can find experiences with foreign accreditation destined to ensure educational quality. An example is the Universidad de San Martin de Porres, whose entire careers have been internationally accredited.

\section{Key words}

Quality assurance; Accreditation; Accreditation in Peru; Accreditation in Latin American.

\section{Introducción}

Desde tiempo atrás el hombre ha buscado la mejora continua en la entrega de servicios y productos que ofrezcan satisfacción a su público objetivo; esto se observa en la administración, cuando las grandes empresas comenzaban a mostrar interés, a partir de la II guerra mundial, en entregar productos que cumplieran con características mínimas antes de llegar al público al cual estaba destinado, surgiendo así los primeros conceptos de calidad, la cual era enmarcada exclusivamente en la empresa y el usuario.

Con el paso de los años se incluyó este concepto en diversos aspectos, que además de la entrega de productos de calidad, también se extendiera a los servicios por lo que actualmente se observa que la calidad es un tema que está inmerso en diversas disciplinas, incorporando la calidad dentro de un escenario más extenso que la empresa propiamente (Freeman, 1994).

En la actualidad, las instituciones de educación superior, como las universidades, por cumplir un carácter de universalidad y desarrollar conocimientos que serán los cimientos para el desarrollo de una sociedad, no deben apartarse de este contexto; la calidad en la educación superior es un término que se viene debatiendo e incursionando desde años atrás, por el rol importante que desempeña en el desarrollo humano, la adquisición de nuevos conocimientos y la investigación, por lo que se ha considerado importante que se dirija hacia una propuesta determinada que se oriente a la mejora de esta enseñanza y progreso del docente a este nivel, que sea una motivación para la evolución constante y la 
capacidad para lograr el mayor desarrollo del servicio y la satisfacción de los usuarios. Asegurar la calidad en la formación universitaria habilita el ingreso a un sistema continuo de autoevaluación y evaluación, que permita que la institución reconozca sus debilidades y fortalezas y de esta forma planifique y organice su progreso, y mejoramiento continuo, que abarque todos los aspectos propios del sistema, permitiendo brindar educación de calidad (Rodríguez, 1991).

Para el desarrollo del presente artículo se decidió tomar como referencia las definiciones emitidas por la UNESCO (1998) como la Declaración Mundial sobre la Educación Superior en el siglo XXI que define la calidad de la educación tomando en cuenta categorías de análisis y no solo como empresa y producto, exponiendo que:

La calidad de la enseñanza superior es un concepto pluridimensional que debería comprender todas sus funciones y actividades: enseñanzas y programas académicos, investigación y becas, personal, estudiantes, edificios, instalaciones, equipamiento y servicios a la comunidad y al mundo universitario. Una autoevaluación interna y un examen externo realizados con transparencia por expertos independientes, en lo posible especializados en lo internacional, son esenciales para la mejora de la calidad. (UNESCO, 1998, art. 11).

Así mismo, en el año 2003 en la "Metting of Higher Education Partners" la UNESCO incorporó el término acreditación, definiéndola cómo la confirmación por parte de un organismo externo (agencia del gobierno, organismo regulador ó grupo de expertos profesionales) del cumplimiento de los estándares claves de la calidad, en una institución o programa (traducción libre). Para lograr la unificación de los procesos de enseñanza superior mediante la labor conjunta entre instituciones de educación superior, por medio de la autoevaluación y evaluación, donde se busca la participación activa de los actores afectados que analizan juntos un conjunto de informaciones suficientemente pertinentes, válidas y fiables con el fin de adoptar decisiones de mejora y no simplemente para sancionar (Morles, 1996).

\section{Los orígenes del aseguramiento de la calidad universitaria}

El término aseguramiento de la calidad en educación se remonta a tiempos de la revolución industrial, cuando se crean los sistemas educativos nacionales, buscando no solo evaluar al alumno sino además el desempeño de los docentes y de la institución en sí misma, surgiendo así los sistemas de supervisión gubernamentales en los que se realizaba visitas muchas veces, inopinadas de funcionarios que emitían informes orales o escritos. Recién a finales del siglo XIX se menciona por primera vez el término evaluación y autoevaluación institucional, surgiendo esta tendencia en los Estados Unidos de América al establecerse las primeras agencias de acreditación, creadas gracias a las universidades privadas, quienes dominaban la educación a ese nivel, buscando acreditar la calidad de los egresados de instituciones de educación superior para la continuidad en sus instituciones. A partir de este momento se da inicio a una tendencia a nivel mundial, desarrollándose diferentes modelos orientados al contexto en el que se desarrolla cada uno (Viñas, 1991).

\section{Modelos de calidad y su aplicación en la educación superior en Estados Unidos de América}

En 1887 se inicia en universidades privadas de los Estados Unidos de Norteamérica, la búsqueda de la calidad educativa de los egresados de la educación superior y se crea la 
primera agencia evaluadora y acreditadora "Middle States Asociation", cuyo objetivo era mejorar la calidad de los programas ofrecidos por las instituciones asociadas. Actualmente las agencias acreditadoras continúan siendo privadas y cada gobierno estatal acompañado de agencias de coordinación no gubernamentales, coordina, norma, financia y supervisa la calidad educativa; teniendo como propósito, el asegurar que lo ofrecido en las instituciones académicas hayan alcanzado un nivel aceptable de calidad (El-Khawas, 2001).

Inicialmente este modelo se regía en normas cuantitativas y arbitrarias en algunos casos. A partir de la década de los 50 (del siglo XX) se introduce el informe periódico y se hace énfasis en la evaluación cualitativa, teniendo como propósito reforzar y mantener la calidad de la educación superior, logrando la confianza pública.

Este sistema se basa en su gran dimensión y diversidad en-la cobertura de la educación, puesto que Estados Unidos de Norteamérica cuenta con diversas instituciones educativas que van desde Universidades, hasta pequeñas instituciones de formación profesional; maneja 2 sistemas de acreditación para la institución:

- La acreditación Institucional o regional: evalúa hasta qué nivel la institución ha alcanzado las metas propiamente trazadas y se asegura que continúen con los procesos que lo permitan.

- La especializada o de programas: otorgada a escuelas o programas profesionales ocupacionales, evalúan de acuerdo a las normas fijadas a nivel nacional en la profesión y hasta qué punto la institución cumple con dichas normas.

Según Yzaguirre (2005) es aquí donde se crea el sistema ISO (International Standard Organization) sistema de evaluación por calidad, el cual tiene su base en el "Total Quality Management" (TQM), este sistema evalúa los procesos de un determinado servicio o producción.

En 1986 se creó el primer paquete de normas ISO 9000, dirigido a sistemas de gestión de calidad, creándose en el año 2000 el ISO 9001-2000 el cual tenía fines de certificación y promueve un enfoque basado en procesos que permite desarrollar, implementar y mejorar la eficacia de un sistema de gestión de calidad, aumentando la satisfacción del cliente mediante el cumplimiento de sus requisitos, necesidades y expectativas, mediante el funcionamiento eficaz de la organización, identificando y administrando las distintas actividades desarrolladas entre sí.

Al incluir las normas ISO 9000 en educación se toma en cuenta dimensiones como:

- Eficacia: lograr que el alumno obtenga los conocimientos establecidos en los planes y programas curriculares.

- Competitividad en términos sociales y del propio individuo: implica que los conocimientos impartidos respondan adecuadamente a la necesidad del individuo para desarrollarse como persona íntegra en la sociedad.

- Procesos y medios: se evalúa los medios empleados para lograr que el estudiante desarrolle adecuadamente su proceso de aprendizaje.

En 1987 se crea el modelo Malcolm Baldrige, cuyo objetivo es optimizar los niveles de calidad y poder emplearlos para la planificación, evaluación y formación dentro del sistema de gestión. Utiliza una doble metodología: autoevaluación y evaluación externa.

Una de las características principales de este sistema en las instituciones educativas es que los criterios de evaluación utilizados pueden ser aplicables a todo nivel educativo y tamaño 
de institución, permitiendo que sea esta misma quien determine las metas que debe alcanzarse, sin necesidad que sea un agente externo el que lo señale. Se enfoca en los procesos y en los valores de la cultura organizacional, permitiendo identificar las debilidades de la institución. Propicia el desarrollo de un plan estratégico y el fortalecimiento de una cultura de calidad, lo que permitirá desarrollar un proceso sostenible a mediano y largo plazo.

\section{Modelos de calidad y su aplicación en la educación superior en América Latina}

Desde la década de 1990, la calidad se ha convertido en un tema trascendental en la agenda de la educación superior de América Latina. Diversos grupos de interés han mostrado creciente preocupación por los diferenciados niveles de calidad que evidencian los sistemas de educación superior nacionales de la región y sus consecuencias, entre ellas, el acceso a la educación superior y el nivel de incertidumbre social acerca de las certificaciones profesionales. Como respuesta a esta preocupación, se pusieron en marcha diversas prácticas de evaluación y acreditación de la calidad de la educación superior en toda la región, tanto para promover el mejoramiento institucional, como para asegurar a la sociedad que las universidades cumplían ciertos estándares de calidad.

El primer país latinoamericano que inició las prácticas de regulación y evaluación de la calidad de la educación superior por iniciativa estatal fue Chile, en 1990 a través del Consejo Superior de Educación - CSE, hoy Comisión Nacional de Acreditación - CNA. Le siguieron el Consejo Nacional de Acreditación - CNA de Colombia en 1992, la Comisión Nacional de Acreditación de Carreras Universitarias - CNACU de Bolivia en 1994, la Comisión Nacional de Evaluación de Evaluación y Acreditación Universitaria - CONEAU de Argentina en 1995, el Consejo de Evaluación, Acreditación y Aseguramiento de la Calidad de la Educación Superior - CEAACES de Ecuador en el año 2000, el Sistema de Evaluación y Acreditación - SEA de Venezuela en 2001, la Agencia Nacional de Evaluación y Acreditación de la Educación Superior - ANEAES de Paraguay en 2003, el Sistema Nacional de Evaluación de la Educación Superior - SINAES de Brasil en 2004, y el Sistema Nacional de Evaluación, Acreditación y Certificación de la Calidad Educativa - SINEACE de Perú en 2006 (Inga y Velásquez, 2005).

Es importante destacar las iniciativas regionales, como el sistema ARCU-SUR (Acreditación regional de carreras universitarias para el MERCOSUR), el SICEVAES (Sistema Centroamericano de Evaluación y Acreditación de la Educación Superior) y la creación de RIACES (Red Iberoamericana para la Acreditación de la Calidad de la Educación Superior).

De acuerdo al último informe del Centro Interuniversitario de Desarrollo (CINDA), editado por Lemaitre y Zenteno (2012), "los procesos de aseguramiento de la calidad en América Latina, muestran una gran diversidad de modelos, los que han ido desarrollándose en función de las necesidades de los respectivos sistemas de educación superior y las características de la cultura local" (pp. 33-34).

Entre las características más importantes de la mayoría de los sistemas de aseguramiento de la calidad en América Latina, destacan que son de iniciativa estatal, aunque con distintos grados de autonomía y descentralización, cuyo propósito principal es dar garantía pública de la calidad mediante la instalación de mecanismos de acreditación de carreras, los cuales están basados en procesos de autoevaluación desarrollados por la carrera o institución y validados por un proceso de evaluación externa. En general, los sistemas latinoamericanos enfatizan la acreditación de carreras o programas de pregrado (llamados normalmente "de grado" en la región), conducentes a un primer grado académico o título habitual. 
Finalmente, los procesos de acreditación son voluntarios en países como Chile y Colombia, y obligatorio en países como Argentina (carreras de interés público y posgrado) y Perú (carreras de salud, educación y derecho) (Lemaitre y Zenteno, 2012).

\section{La experiencia peruana}

Desde mediados de los noventa se realizan en el Perú, acciones orientadas a establecer mecanismos de aseguramiento de la calidad en la educación superior universitaria. Pero es recién a mediados de la década del 2000 que se crea oficialmente un sistema nacional con este fin (Nava, 2004; López, Soria, 2004; CAFME, 2003). A continuación se describe brevemente este proceso:

Cuadro resumen 1. Desarrollo de los mecanismos de aseguramiento de la calidad de la Educación Superior en el Perú

\begin{tabular}{|c|c|c|c|c|}
\hline Año & Institución/Organización & Propósito & Aportes & Vigencia \\
\hline 1995 & $\begin{array}{l}\text { Consejo Nacional para la } \\
\text { Autorización de } \\
\text { Funcionamiento de } \\
\text { Universidades (CONAFU). }\end{array}$ & $\begin{array}{l}\text { Regular el } \\
\text { funcionamiento de las } \\
\text { nuevas universidades. }\end{array}$ & $\begin{array}{l}\text { Autoriza el } \\
\text { funcionamiento } \\
\text { permanente de las } \\
\text { universidades. }\end{array}$ & Actual \\
\hline 1999 & $\begin{array}{l}\text { Comisión para la Acreditación } \\
\text { de Facultades o Escuelas de } \\
\text { Medicina, CAFME. }\end{array}$ & $\begin{array}{l}\text { Responder a la presión } \\
\text { del crecimiento } \\
\text { desmesurado de } \\
\text { facultades de medicina } \\
\text { en el Perú y a la } \\
\text { decreciente calidad en } \\
\text { la formación en } \\
\text { algunas de ellas. }\end{array}$ & $\begin{array}{l}\text { Estándares mínimos } \\
\text { para la acreditación de } \\
\text { Facultades/Escuelas de } \\
\text { Medicina. } \\
\text { Procedimientos para la } \\
\text { acreditación. } \\
\text { Verificadores } \\
\text { capacitados. } \\
\text { Todas las } \\
\text { Facultades/Escuelas de } \\
\text { Medicina incorporadas al } \\
\text { proceso. }\end{array}$ & Hasta el 2006 \\
\hline 1999 & $\begin{array}{l}\text { Consorcio de Universidades } \\
\text { (formado por cuatro } \\
\text { universidades privadas). }\end{array}$ & $\begin{array}{l}\text { Aportar al desarrollo y } \\
\text { mejora institucional } \\
\text { mediante la } \\
\text { autoevaluación del } \\
\text { proceso formativo. }\end{array}$ & $\begin{array}{l}\text { "Modelo para promover } \\
\text { la cultura de la } \\
\text { autoevaluación". } \\
\text { Promoción de la } \\
\text { autorregulación y el } \\
\text { mejoramiento continuo. }\end{array}$ & Actual \\
\hline 2000 & $\begin{array}{l}\text { Varias universidades que } \\
\text { ofrecen especialidades en } \\
\text { ingeniería, con el apoyo de la } \\
\text { Accreditation Board for } \\
\text { Engineering and Technology } \\
\text { (ABET). }\end{array}$ & $\begin{array}{l}\text { Asegurar la calidad de } \\
\text { la formación en } \\
\text { ingeniería. }\end{array}$ & $\begin{array}{l}\text { Inició la acreditación } \\
\text { internacional de las } \\
\text { carreras de ingeniería en } \\
\text { el Perú. }\end{array}$ & Actual \\
\hline 2002 & $\begin{array}{l}\text { Comisión Nacional de } \\
\text { Rectores para la Acreditación } \\
\text { (Asamblea Nacional de } \\
\text { Rectores, ANR). }\end{array}$ & $\begin{array}{l}\text { Observar e informar } \\
\text { sobre los procesos de } \\
\text { mejora continua, } \\
\text { autoevaluación y } \\
\text { acreditación de las } \\
\text { universidades. } \\
\text { Hacer propuestas de } \\
\text { estándares mínimos de } \\
\text { acreditación para las } \\
\text { carreras profesionales } \\
\text { que ofrecen las } \\
\text { universidades }\end{array}$ & $\begin{array}{l}\text { “Modelo de } \\
\text { Autoevaluación con } \\
\text { fines de Mejora de las } \\
\text { Carreras Universitarias" } \\
\text { Apoyo a universidades } \\
\text { en sus procesos de } \\
\text { autoevaluación y } \\
\text { mejora. } \\
\text { Promovió la creación de } \\
\text { instancias especialmente } \\
\text { dedicadas a calidad y } \\
\text { acreditación en las }\end{array}$ & $\begin{array}{l}\text { Hasta el } 2007 \\
\text { en esta } \\
\text { actividad. }\end{array}$ \\
\hline
\end{tabular}




\begin{tabular}{|c|c|c|c|c|}
\hline & & peruanas. & universidades. & \\
\hline 2004 & $\begin{array}{l}\text { Asociación Peruana de } \\
\text { Facultades y Escuelas de } \\
\text { Enfermería, ASPEFEEN. }\end{array}$ & $\begin{array}{l}\text { Asegurar la calidad de } \\
\text { la formación en } \\
\text { enfermería }\end{array}$ & $\begin{array}{l}\text { Estándares básicos y de } \\
\text { calidad. } \\
\text { Capacitación en } \\
\text { autoevaluación. } \\
\text { Formación de } \\
\text { evaluadores externos. } \\
\text { Proceso piloto de } \\
\text { autoevaluación y } \\
\text { evaluación externa. }\end{array}$ & $\begin{array}{l}\text { Hasta el } 2007 \\
\text { en esta } \\
\text { actividad. }\end{array}$ \\
\hline 2006 & $\begin{array}{l}\text { Asociación Peruana de } \\
\text { Facultades y Escuelas de } \\
\text { Obstetricia, ASPEFOBST. }\end{array}$ & $\begin{array}{l}\text { Asegurar la calidad de } \\
\text { la formación en la } \\
\text { carrera de obstetricia. }\end{array}$ & $\begin{array}{l}\text { Currículo base de la } \\
\text { carrera de obstetricia. } \\
\text { Estándares de calidad. } \\
\text { Guía de autoevaluación. } \\
\text { Capacitación en } \\
\text { autoevaluación. } \\
\text { Formación de } \\
\text { evaluadores externos. } \\
\text { Proceso piloto de } \\
\text { autoevaluación y } \\
\text { evaluación externa. }\end{array}$ & $\begin{array}{l}\text { Hasta el } 2007 \\
\text { en esta } \\
\text { actividad. }\end{array}$ \\
\hline 2006 & $\begin{array}{l}\text { Sistema Nacional de } \\
\text { Evaluación, Acreditación y } \\
\text { Certificación de la Calidad } \\
\text { Educativa, SINEACE. }\end{array}$ & $\begin{array}{l}\text { Garantizar a la } \\
\text { sociedad que las } \\
\text { instituciones de } \\
\text { educación de todo el } \\
\text { país ofrezcan un } \\
\text { servicio de calidad. }\end{array}$ & $\begin{array}{l}\text { Consolida la existencia } \\
\text { de un sistema nacional } \\
\text { de aseguramiento de la } \\
\text { calidad educativa en el } \\
\text { Perú. }\end{array}$ & Actual \\
\hline
\end{tabular}

Entretanto, algunas universidades iniciaron procesos tendientes a lograr acreditaciones por entidades extranjeras, con el fin de elevar su prestigio y el reconocimiento de sus egresados. EI CNA de Colombia y la Red Internacional de Evaluadores, RIEV, además de ABET, estuvieron entre las primeras. Más adelante, empezaron a recurrir a otras organizaciones de los Estados Unidos de Norteamérica y de Europa.

\section{La experiencia en las carreras de la salud}

Las carreras profesionales de Obstetricia y Enfermería iniciaron procesos orientados al aseguramiento de la calidad en la formación. Ambas organizadas en sendas asociaciones, la Asociación Peruana de Escuelas y Facultades de Obstetricia (ASPEFOBST) y la Asociación Peruana de Facultades y Escuelas de Enfermería (ASPEFEEN); además de Medicina, que realizó este proceso a través de las siguientes organizaciones: Ministerio de Salud-Colegio Médico del Perú-Asociación Peruana de Facultades de Medicina (ASPEFAM).

La experiencia desarrollada fue la siguiente:

\section{ASPEFOBST:}

- 1996-1999: Actualización del perfil profesional del licenciado en obstetricia, proceso realizado descentralizado y participativamente.

- 2003: Levantamiento de información, principalmente en las regiones donde se formaban profesionales en obstetricia, con el fin de analizar la situación de la salud materna perinatal para, en base a esa información y de la revisión de los currículos de estudio de diversas Facultades y Escuelas, construir un currículo base para la formación en obstetricia. 
- 2005: Currículo base concluido y validado a través de su implementación en Facultades/ Escuelas piloto.

- 2006: Contando ya con un currículo base homologado y un perfil del egresado, se inicia la construcción de los estándares de calidad para la acreditación, los que expresan objetivos a alcanzar en un nivel óptimo.

- 2006-2007: Estándares validados. Capacitación de evaluadores de universidades de todo el país. Inicio de procesos de autoevaluación y conformación de equipos de evaluadores pares para la evaluación externa, proceso que se llevó a cabo durante varios años.

\section{ASPEFEEN}

- 1998-1999: Formulación del perfil educativo de las egresadas de los Programas de Pregrado de las Facultades/Escuelas de Enfermería, en términos de competencias.

- 2003-2004: Construcción de estándares básicos de calidad para la acreditación (utilizan el modelo colombiano)

- 2005-2006: Capacitación de equipos de autoevaluación. Inicio de procesos de autoevaluación. Formación en autoevaluación y capacitación de evaluadores externos.

- 2006-2007: Visitas de evaluación externa. Proceso que continúa durante varios años.

\section{EI SINEACE}

La vigente Ley Universitaria $\mathrm{N}^{\circ} 23773$, no precisa la aplicación de prácticas de evaluación y acreditación de la calidad para las instituciones de educación superior. A fin de incorporar estas prácticas, la Ley General de Educación Nº 28044 promulgada en el año 2003 establece y garantiza el funcionamiento del Sistema Nacional de Evaluación, Acreditación y Certificación de la Calidad Educativa. Y en el 2006, mediante Ley $N^{\circ} 28740$ se aprueba la Ley del Sistema Nacional de Evaluación, Acreditación y Certificación de la Calidad Educativa SINEACE.

EI SINEACE tiene la finalidad de garantizar a la sociedad que las instituciones de educación de todo el país ofrezcan un servicio de calidad, recomendando aplicar acciones para superar las debilidades y carencias identificadas en los resultados de las autoevaluaciones y evaluaciones externas. EI SINEACE tiene un ente rector que es el Consejo Superior, el cual es un organismo público descentralizado, adscrito al Ministerio de Educación, y tiene autonomía normativa, administrativa, técnica y financiera. A su vez, tiene órganos operadores, encargados de garantizar la calidad educativa en el ámbito de la educación básica y técnica productiva a través del Instituto Peruano de Evaluación, Acreditación y Certificación de la Calidad de la Educación Básica - IPEBA, en la educación superior no universitaria a través del Consejo de Evaluación, Acreditación y Certificación de la Calidad de Educación Superior No Universitaria - CONEACES y en la educación superior universitaria a través del Consejo de Evaluación, Acreditación y Certificación de la Calidad de la Educación Superior Universitaria - CONEAU. En el contexto de la educación superior, el CONEAU es el órgano operador encargado de definir los criterios, indicadores y estándares de medición para garantizar en las universidades públicas y privadas los niveles aceptables de calidad, así como alentar la aplicación de medidas requeridas para su mejoramiento (Ley $\mathrm{N}^{\circ} 28740$, 2006).

A la fecha, el CONEAU ha acreditado solo a 14 carreras de nueve universidades, entre públicas y privadas. No obstante, diversas carreras universitarias han sido acreditadas por organismos internacionales. La ley establece que la única acreditación válida es la entregada por el SINEACE, a través del CONEAU. Pese a ello, el CONEAU permite que 
aquellas carreras acreditadas por organismos extranjeros puedan ser aprobadas, luego de un proceso de validación (CONEAU, 2014).

\section{La experiencia en la Universidad de San Martín de Porres, USMP}

Desde inicios del milenio, la política de la USMP, estuvo orientada a asegurar la calidad de los procesos internos, como rasgo distintivo de sus actividades académicas y administrativas.

El proceso llevado a cabo por la USMP, puede ser resumido de la siguiente forma:

- 2003:

- Se crea la Unidad de Autoevaluación y Acreditación, orientada a conducir el proceso de Autoevaluación y a asesorar en los futuros procesos de Acreditación.

- Se diseña el documento de trabajo "Estándares de Autoevaluación y Acreditación", que serviría de instrumento para evaluar las carreras profesionales de la Universidad

- 2004-2005: Se realiza la sensibilización del documento de trabajo, impulsando los procesos de autoevaluación, así como el acompañamiento a las Facultades en este proceso.

- 2006:

- Las comisiones de autoevaluación y acreditación instaladas en cada facultad, concluyen los procesos de autoevaluación de sus carreras profesionales y elaboran los respectivos informes y diseñan sus planes de mejora.

- La Facultad de Ciencias Administrativas y Recursos Humanos y la de Ciencias Contables, Económicas y Financieras establece coordinaciones con la Asociation of Collegiate Business School an Programs (ACBSP). Luego de cumplir con los requerimientos exigidos, las carreras de la Facultad de Ciencias Administrativas y Recursos Humanos, obtuvieron la mencionada acreditación.

- 2007-2013:

- La Universidad orienta sus esfuerzos hacia la acreditación internacional.

- Se acreditan internacionalmente las primeras carreras profesionales: Ciencias de la Comunicación (periodismo), por el Consejo Latinoamericano de Acreditación de la Educación en Periodismo (CLAEP), y Turismo y Hotelería por la Tourism Education Quality (TEDQUAL), organismo de la Organización Mundial de Turismo.

- La Universidad inicia un acercamiento con la Comisión on Colleges de la Southern Association of Colleges and Schools (SACSCOC) y empieza procesos de acreditación de las carreras profesionales de Ingeniería, con la Accreditation Board for Engineering and Technology (ABET).

- Durante el 2008 consolida sus instrumentos de gestión de la calidad a través del diseño, formulación y aprobación de la normatividad necesaria.

- Continúan los sus procesos de autoevaluación, así como con los procesos de acreditación internacional.

- La Facultad de Medicina acredita internacionalmente con la RIEV

- La Facultad de Derecho es acreditada por la "Agència per a la Qualitat del Sistema Universitari a Catalunya - AQU” de España.

- El 2010, la USMP, establece objetivos orientados a consolidar la calidad educativa y a fines de ese mismo año se inicia un acercamiento a la Axencia para a Calidade do Sistema Universitario de Galicia (ACSUG). 
- Son acreditadas por ACSUG, las carreras profesionales de Obstetricia, Enfermería, Odontología, Medicina, Psicología y el Instituto para la Calidad Educativa.

- En el 2013 la USMP, tiene 16 escuelas profesionales acreditadas internacionalmente.

Cuadro resumen 2. Consolidado del proceso de acreditación de la USMP

\begin{tabular}{|c|c|c|}
\hline Facultad & Agencia & $\begin{array}{l}\text { Acreditada hasta el } \\
\text { año }\end{array}$ \\
\hline $\begin{array}{l}\text { Facultad de Ciencias } \\
\text { Administrativas y Recursos } \\
\text { Humanos }\end{array}$ & $\begin{array}{l}\text { Association of Collegiate Business Schools and } \\
\text { Programs - ACBSP } \\
\text { European Council For Business Education- ECBE }\end{array}$ & $\begin{array}{l}2019 \\
2015\end{array}$ \\
\hline $\begin{array}{l}\text { Facultad de Ciencias } \\
\text { Contables, Económicas y } \\
\text { Financieras }\end{array}$ & $\begin{array}{l}\text { Association of Collegiate Business Schools and } \\
\text { Programs - ACBSP } \\
\text { European Council For Business Education- ECBE }\end{array}$ & $\begin{array}{l}2019 \\
2015\end{array}$ \\
\hline $\begin{array}{l}\text { Facultad de Ciencias de la } \\
\text { Comunicación, Turismo y De } \\
\text { Psicología }\end{array}$ & $\begin{array}{l}\text { Consejo Latinoamericano de Acreditación en la } \\
\text { enseñanza del Periodismo- CLAEP } \\
\text { Tourism Education Quality- TEDQUAL } \\
\text { Axencia para a Calidade do Sistema Universitario de } \\
\text { Galicia - España- ACSUG }\end{array}$ & $\begin{array}{l}2016 \\
2016 \\
2018\end{array}$ \\
\hline Facultad de Derecho & $\begin{array}{l}\text { I'Agéncia per a la Qualitat del Sistema Universitari a } \\
\text { Catalunya- AQU }\end{array}$ & 2014 \\
\hline $\begin{array}{l}\text { Facultad de Ingeniería y } \\
\text { Arquitectura }\end{array}$ & $\begin{array}{l}\text { Accreditation Agency for Degree Programmes } \\
\text { In Engineering (ASIIN) } \\
\text { European Accreditation of Engineering } \\
\text { Programmes (EUR-ACE) } \\
\text { Accreditation Board for Engineering and } \\
\text { Technology -ABET } \\
\text { Axencia para a Calidade do Sistema Universitario de } \\
\text { Galicia - España- ACSUG }\end{array}$ & $\begin{array}{l}2015 \\
2015 \\
2016\end{array}$ \\
\hline $\begin{array}{l}\text { Facultad de Medicina } \\
\text { Humana }\end{array}$ & $\begin{array}{l}\text { Red Internacional de Evaluadores- RIEV } \\
\text { Agencia para la Calidad del Sistema Universitario de } \\
\text { Galicia- ACSUG }\end{array}$ & $\begin{array}{l}2014 \\
2018\end{array}$ \\
\hline $\begin{array}{l}\text { Facultad de Obstetricia y } \\
\text { Enfermería }\end{array}$ & $\begin{array}{l}\text { Agencia para la Calidad del Sistema Universitario de } \\
\text { Galicia- ACSUG }\end{array}$ & 2017 \\
\hline Facultad de Odontología & $\begin{array}{l}\text { Instituto Latinoamericano de Acreditación en } \\
\text { Odontología (ILAO) } \\
\text { Agencia para la Calidad del Sistema Universitario de } \\
\text { Galicia- ACSUG }\end{array}$ & 2015 \\
\hline $\begin{array}{l}\text { Instituto para la Calidad de } \\
\text { la Educación }\end{array}$ & $\begin{array}{l}\text { Agencia para la Calidad del Sistema Universitario de } \\
\text { Galicia- ACSUG }\end{array}$ & 2018 \\
\hline
\end{tabular}

\section{Experiencia en acreditación en la Facultad de Obstetricia y Enfermería. FOE}

Luego de solicitar la evaluacion externa a diferentes agencias acreditadoras del mundo y no lograr alguna que acreditara carreras de la salud, fuera de su ámbito geográfico, se logró que en el año 2009 la Agéncia per a la Qualitat del Sistema Universitari a Catalunya (AQU), acepte la solicitud para evaluar a sus carreras profesionales del pregrado y del posgrado.

La FOE analizó el modelo de AQU, avanzando en el análisis de los primeros estándares de la guía de trabajo, sin embargo la agencia decidió retirarse de América Latina por la atención del programa “Docentia” en España. 
Por recomendación de AQU, la FOE empieza a gestionar un acercamiento institucional con la Axencia para a Calidade do Sistema Universitario de Galicia (ACSUG), institución que pertenece y sigue los lineamientos del Espacio Europeo de Educación Superior (EEES) y lo establecido por la European Association for Quality Assurance in Higher Education (ENQA).

En el año 2011 la FOE es acreditada por ACSUG por el máximo plazo otorgado por dicha institución, 6 años. ACSUG certifica que la Facultad de Obstetricia y Enfermería de la USMP cumple con los estándares europeos para la garantía interna de la calidad de centros de educación superior. Este reconocimiento incluye a sus carreras de pregrado y del posgrado, así como a sus sedes y filiales.

Consecuentemente, el compromiso asumido por la comunidad universitaria de la FOE, es proseguir en la senda de la mejora continua de la calidad e incorporar a la autoevaluación en su cultura institucional.

\section{Discusión y conclusiones}

Es destacable el interés y la voluntad del Estado peruano y de las propias instituciones de educación superior, por establecer mecanismos que aseguren la calidad educativa en la formación universitaria, sea a través del sistema nacional o de referentes internacionales. La sociedad debe tener la garantía de que la formación de profesionales contribuirá al desarrollo del país y esa debe ser la meta a alcanzar.

\section{Referencias}

Comisión de Acreditación de Facultades o Escuelas de Medicina Humana, CAFME (2003) Informe sobre el proceso de Acreditación de Facultades o Escuelas de Medicina (CAFME, Comunicado 002). Perú, Lima.

Comisión Nacional de Evaluación y Acreditación Universitaria, CONEAU (2014). Acreditación de carreras de Pregrado. Recuperado de: http://www.coneau.gob.pe/acreditacion/2013-01-10-14-54-32/2013-01-10-15-13-14.html

El-Khawas, E. (2001). Accreditation in the USA: origins, developments and future prospects. (UNESCO, Informe). Recuperado de: http://unescodoc.unesco.org/images/o012/129295e.pdf

Freeman, R. (1994). Quality Assurance in Secondary Education. Quality assurance in Education, 2(1), 21-25. Recuperado de:

http://www.emeraldinsight.com/journals.htm?articleid=839508\&show=abstract

Inga, M.G. y Velásquez, D. (2005). La evaluación y acreditación de la calidad en las leyes de educación superior de América Latina. Perú, Lima: Universidad Nacional Mayor de San Marcos, Comisión de Coordinación de Reforma Universitaria.

Leimaitre, M.J. y Zenteno, M.E. (eds.) (2012). Aseguramiento de la calidad en Iberoamérica. Educación Superior (CINDA Informe 2012). Recuperado de: http://www.cinda.cl/download/libros/Aseguramiento\%20de\%20la\%2ocalidad\%20en\%2 olberoam\%C3\%Agrica\%202012.pdf

Ley $\mathrm{N}^{\circ}$ 28740. Ley del Sistema Nacional de Evaluación, Acreditación y Certificación de la Calidad Educativa - SINEACE (2006). 
López Soria, J.I. (2004). Calidad y acreditación universitaria. Revista Iberoamericana de Educación, OEI, 35, 113-132. Recuperado de: http://www.rieoei.org/index.php

Morles, V. (1996). La experiencia internacional sobre evaluación y acreditación de la educación superior y de postgrado: una visión panorámica. Educación Superior y Sociedad, 7(1), 59-72. Recuperado de: http://ess.iesalc.unesco.org.ve/index.php/ess/article/view/270/225

Nava, H. (2004). Evaluación y acreditación de la educación superior: El caso del Perú. En Instituto Internacional para la Educación Superior en América Latina y el Caribe (ed.), La Evaluación y la Acreditación de la Educación Superior en América Latina y el Caribe [Versión Adobe Digital Editions] (pp.209-224). Recuperado de: http://unesco.org.ve

Organización de las Naciones Unidas para la Educación, la Ciencia y la Cultura, UNESCO (1998). Declaración Mundial sobre la Educación Superior en el siglo XXI: visión y acción. Recuperado de: http://unesco.org

Organización de las Naciones Unidas para la Educación, la Ciencia y la Cultura, UNESCO. (2004). Metting of Higher Education Partners (UNESCO, Final report). Recuperado de: http://unesdoc.unesco.org/images/0013/001352/135213e.ppdf

Reglamento de la Ley $N^{\circ}$ 28740, Ley del Sistema Nacional de Evaluación, Acreditación y Certificación de la Calidad Educativa (2007).

Rodríguez, S. (1991). Calidad universitaria: un enfoque institucional y multidimensional. Madrid: Consejo de Universidades. Secretaría General. Recuperado de: http://dialnet.unirioja.es/servlet/articulo?codigo $=2100413$

Viñas, J.A. (1991). La Universidad norteamericana, el autoestudio y la acreditación. Un modelo de mejoramiento académico. Publicaciones ANUIES, 79, 1-9. Recuperado de: file:///C:/Users/bibatenciono6/Downloads/Revista79_S1A2ES\%20(1).pdf

Yzaguirre, L.E. (2005). Calidad educativa e ISO 9001-2000 en Mexico. Revista Electrónica Iberoamericana sobre Calidad, Eficiencia y Cambio en Educación, 3(1). Recuperado de: http://www.rieoei.org/deloslectores/945Yzaguirre.PDF 


\section{Autores}

Ana Miluzka Baca Gamarra

Licenciada en Obstetricia. Magister en Salud Sexual y Reproductiva.

Coordinadora adjunta del Centro especializado en salud sexual y reproductiva del adolescente -Facultad de Obstetricia y Enfermería de la Universidad de San Martín de Porres. Área de experiencia e investigación: Salud sexual y reproductiva, adolescentes, calidad de atención

Facultad de Obstetricia y Enfermería. Universidad de San Martin de Porres. Av. Salaverry N 1136 Jesús María, Lima, Perú. Teléfono.471-1171_abacag@usmp.pe

María Elvira León Di Gianvito

Licenciada en Psicología. Presidenta del Instituto Peruano de Paternidad Responsable, Inppares. Coordinadora del Comité de Acreditación y Calidad de la FOE, USMP. Áreas de experiencia e investigación: calidad educativa; gestión de la acreditación universitaria; certificación de competencias profesionales; desarrollo de capacidades en adultos; salud sexual y salud reproductiva

Facultad de Obstetricia y Enfermería. Universidad de San Martín de Porres . Av. Salaverry No 1136 Jesús María, Lima, Perú. Teléfono.471-1171 - mleond@usmp.pe

Juan José Mayta Carlos

Licenciado en Ingeniero de Sistemas. Coordinador de la Unidad de Sistemas de la Facultad de Obstetricia y Enfermería de la Universidad de San Martín de Porres. Área de experiencia e investigación: gestión de sistemas de información y mejora de procesos

Facultad de Obstetricia y Enfermería. Universidad de San Martín de Porres . Av. Salaverry No 1136 Jesús María, Lima, Perú. Teléfono.471-1171 - jmaytac@usmp.pe

Carlos Bancayán Oré

Licenciado en Sociología. Maestría en Gestión de la Calidad, Autoevaluación y Acreditación. Doctor en Educación. Coordinador Académico y docente de la Universidad de San Martín de Porres. Áreas de experiencia e investigación: calidad educativa; gestión de la acreditación universitaria

Estudios Generales. Universidad de San Martín de Porres. Av. Las Calandrias s/n Santa Anita, Lima - Perú. Teléfono: 362-0064 - cbancayano@usmp.pe 